restless, perpetually turning from side to side in bed, unable to answer questions, the only reply to attempts to arouse her being an appeal for water. Hæmatemesis was frequent and the uterine hæmorrhage copious. On examination a remarkable condition was disclosed. Below the waist-line more than half of the total skin area was the seat of enormous subcutaneous bæmorrhages. The front and back of each thigh, both buttocks, the greater part of the left leg and the right foot presented continuous patches, dark purple in colour, with sharply-defined and often crescentic margins. The intervening skin, as elsewhere throughout the body, was normal. There was no jaundice. No satisfactory examination of the abdomen could be made owing to the pain and the girl's restless condition. There was incontinence of urine. The bowels were not moved. The temperature was subnormal, respirations 48 , and pulse 120 and very feeble. She was admitted into hospital under Dr. E. F. Trevelyan, but died at 7.40 P.M. - less than three and a half days after taking the poison.

Necropsy. - The post-mortem examination was made 19 hours later. The body was that of a heavily-built stout girl, the abdominal fat being at least one and a half inches in thickness. No secretion could be squeezed from the mammæ. The large purpuric areas were already showing signs of putrefaction, the edges becoming green, and on the right thigh there were a few bullæ filled with blood-stained fluid. It was noted that there was considerable staining around the puncture made by a hypodermic needle. The brain was normal. On opening the thorax it was found that the pleural cavities contained a small quantity of dark-coloured fluid. Much hæmorrhage had occurred into the mediastinal tissues. Numerous subpleural ecchymoses were seen. The heart and lungs were normal. There was slight excess of peritoneal fluid hæmorrhagic in character. Most extensive retroperitoneal hæmorrhages were present, especially about the neighbourhood of the left kidney, while there were also hæmorrhages in the omentum and the mesentery. The liver was enlarged, weighing 54 ounces, and was of a brilliant canary-yellow colour. The liver substance bulged from its capsule on section and presented the appearance of acute fatty degeneration. There were hæmorrhages into the wall of the gall-bladder, which was thickened and gelatinous-looking. The kidneys were normal in size and on section pale yellow in colour. The stomach was filled with dark fluid material; its mucous membrane appeared normal. The uterus was enlarged and contained a hæmorrhagic mole consistent with the history of a two months pregnancy. The right ovary showed a corpus luteum. The rest of the abdominal viscera were normal in appearance. Unfortunately no specimen of urine was obtained.

Sir Thomas Oliver, who has examined microscopic specimens from the case, writes: "The liver shows signs of very advanced fatty degeneration, a large number of the cells having been completely transformed. The kidney shows very slight changes, indicating, therefore, that the brunt of the poison had been borne by the liver. The fatty degeneration so marked in character is interesting, seeing the short time the patient lived after taking the poison."

Remarks.-Jaundice due to blocking of the bile-capillaries is present in 80 per cent. of these cases by the third day. Though its early appearance is of serious import, speaking generally, it bears no constant relationship to the gravity of the disease or to the extent of the changes in the liver. It may well be that in some rapidly fatal cases, such as this, death comes too early for jaundice to develop. As is usual the primary symptoms - those of gastro-intestinal irritationwere marked off from the secondary signs of toxic poisoning due to hepatic failure by a period of comparative comfort on the beginning of the third day. The only symptom which did not share in this improvement was the thirst which throughout the illness was persistent and distressing. We think it likely that the massive subperitoneal hæmorrhages stood in direct causal relationship to the abdominal pain of the latter part of the illness, and we would compare this with similar conditions as they occur in Henoch's purpura and in acate hæmorrhagic pancreatitis. The subcutaneons bleedings in their wide extent were unlike anything seen in ordinary eases of purpura, and, so far as we know, have never before been recorded in phosphorus poisoning.

Leeds.

\section{FRIEDREICH'S ATAXIA.}

\author{
By A. ROWLEY MOODY, M.B., C.M. EDIN.
}

Friedreich's ataxia is a sufficiently rare disease for an account of a series in one family to be interesting, with the possibility of adding something to our knowledge of the disease or the means of successfully dealing with it.

I was consulted on May 25th, 1899, by a young woman, aged 22 years. Her father died from pneumonia when 64 years old. He had been a strong, vigorous man, and the mother, who accompanied the patient, was strong and well. Menstruation began at the age of 18 years, and for a time she was quite regular and normal in that respect, but had not been unwell for 18 months prior to the time she first consulted me. She said that she had lost a good deal of weight, did not sleep in the early part of the night, was subject to dreams, and was not refreshed in the morning. Her mother said she twitched and talked in her sleep, and that she was a good deal upset mentally, often brooding over the disease which she believed she had inherited, and which she thought was attacking her.

The patient had noticed an increasing difficulty in getting about for six or seven years and was afraid she was going like her sisters and brother. She first noticed that she was clumsy and easily tripped up and could not run. She stood with feet apart and turned somewhat inwards, with the body and head inclined forward. The gait was unsteady and clumsy and zig-zag. The unsteadiness was slightly increased by closing the eyes, but markedly so on turning, so much so that she had to clutch at something fixed; occasionally jerky and irregular movements took place in the legs, arms, and neck. These symptoms, the mother said, were more pronounced in bed at night.

On asking the patient to pick up a pencil the hand and arm, instead of coming in one continuous line to the object, made several jerky, hovering movements as if uncertain of their aim or wishing to put the object off guard that it was intended to seize. Eventually she took it with a clutching, hurrying grasp. The front part of the foot had to be raised by bending the knee more than usual to clear the floor, but there was no thumping down of the heels. There was some delay in commencing any movement. Mentally she was alert and intelligent, but the face was expressionless except for the eyes. At the same time she was able to laugh and to smile, but it suggested an effort to do so. The speech was slow, hesitating, and delivered in as few words as possible with a monotonous scanning intonation. There was great delay in commencing replies to questions, so much so that I thought at first she did not intend to answer at all. There was well-marked lateral and antero-posterior curvature of the spine.

There were no areas of anæsthesia, no neuralgia, no crises. The pupils reacted normally for light and distance. There was no optic atrophy; the sight remained still good. There was no nystagmus at that time. There was complete normal control of the bladder and the other organic reflexes were normal. The knee-jerk was absent in the left leg, but there was a slight movement on the right side. The hands were not deformed, but hung listlessly in her lap or by her side ; the feet were turned a little inwards.

In view of the bent condition of the spine and the evident difficulty in keeping herself erect, as well as to alleviate any irritation in the cord which would be prodnced or increased by this altered position, a well-fitting poroplastic jacket was applied, and in consideration of the probable developmental origin of the disease she was put upon a course of thyroid tabloids, and in the course of a few years ovarian tabloids were added. Both of these tabloids she has continued intermittently up to the present time.

I examined her recently and found there was still the same incoördination, but the paresis had not appreciably increased in the legs or the arms. The knee-jerks were completely absent in both legs. There was no deformity of hands or feet. She was no thinner. Nystagmus was present on turning the eyes in a constrained position. The speech has remained the same in general character, but now there is no delay in replying, and there is more animation. The expression of the face and the attitude are as first described.

On going into the family history I found her family were 
yeomen farmers, and for several hundred years had owned and lived at their farm. This is situated in one of the healthiest districts in the kingdom, where the people are notoriously robust and hardy. They have records of several intermarriages amongst relatives, probably brought about by the desire to keep the property in the family. The father and mother were second cousins, and were much above the average in physique and strength. The mother says her grandmother suffered in the same way as these children. There were nine children, five of whom are healthy and four developed this disease.

The eldest child, a girl, was said to commence the disease at the age of 14, and died from it at the age of 26 , being then unable to leave her chair to which she was lifted out of bed. The feet and hands, they say, were deformed in the same way as the two cases that follow.

The eldest son commenced to show symptoms between 13 and 14 years of age. When I saw him he was a man of enormous physique, 6 feet 2 inches in height, and broad in proportion. He was helped from bed every morning into a specially made massive and immovable chair where he remained all day. His mental powers were good, his speech and facial aspect were as described in the first case, and the eyes were normal; there was complete paresis of the legs with great stiffness, and the legs were swollen to an enormous size, with the skin coarse and pitted, almost suggesting elephantiasis. The feet were characteristically deformed, short, stumpy, with high-arched instep, with the toes over-extended at the interphalangeal joints. When I first saw him there were a number of deep excavated foul and painful ulcers in the legs; these, they said, had been caused by slight knocks. They slowly disappeared after prolonged treatment, and as great care was exercised not to knock them there was no recurrence. He said that the least scratch or cut was difficult to heal. The paresis was almost complete in the arms and hands; he could, however, lightly grasp between the index and second fingers; the thumb was entirely useless. The manus cava was well marked. Lateral nystagmus was also present in fixing the eye at an unusual angle. There were no crises, nor neuralgias of any kind. The feet were drawn inwards so that they rested on their outside edges. He died eight zears ago from pneumonia at the age of 42 years.

The next child affected, a girl, showed symptoms first at the age of 14 years. She was able to get about up to the age of 35 , but is now unable to walk or feed herself. She has slight power of movement in the arms and only with great difficulty can grasp a pencil, when it is held up to her, between the index and second fingers. Her feet and hands show the pes cavus and manus cava symptoms in a typical manner. Nystagmus is present. The speech is thick and monotonous and the attitude and facial expression are similar to the cases already described, with well-marked bending of the spine. The eyes are normal and the sight is good. She complains of no pain and says she should be well if she only had the use of her limbs. During the last two years her legs have begun to swell, and the swelling is now well marked, but not extensive, from three inches below the knee downwards. The swelling does not pit easily on pressure and the skin in the affected part is beginning to get coarse, but there are no ulcers.

The second son, although not afllicted with this disease, is mentally deficient. He is, however, a well-grown powerfal man. With this exception, all the children are mentally much above the average and are fine specimens of humanity, one son having a wide reputation as an athlete.

The only case which has been treated for the constitutional disease is the one first described, and in view of the fact that the disease had already existed for nearly eight years and taking into account the age of the patient when treatment was commenced, I think that we may regard the progress of this case as favourable. It is impossible to say that the disease has been arrested ; in fact, in the nystagmus and knee reflexes the disease has made some headway, but, as the history shows, slight improvement has taken place in some respects. Further, the locomotive powers and the use of the arms are quite as good now as they were when I first saw her 11 years ago.

It would be interesting to know the result of continuous treatment of the same kind applied in the early stages of Friedreich's ataxia, especially in young patients in whom active development is still progressing.

The history of these cases strongly supports the theory that the disease is hereditary, and bears out the idea that it may be caused by some error in the developmental forces, which is not accidental and which may possibly arise in some cases from consanguinity. But apart from the interest in the hereditary character of this disease to which this range of cases clearly points, there are some clinical aspects which I have not seen recorded hitherto. CEdema appeared in the three children who had suffered for the greatest number of years and was a symptom which developed late in the course of the disease and which progressed pari passu with the paresis. At the same time the skin also underwent a progressive change, becoming in the first place coarse and pitted and eventually assuming almost the appearance of elephantiasis. In these cases it was associated with trophic ulcers, which, however, were different from the ulcers found in cases of locomotor ataxia and other spinal lesions in that they were markedly painful.

Shelton, Stoke-upon-Trent.

\section{DISPLACEMENTS OF THE SEMILUNAR CARTILAGES.}

By KENELM H. DIGBY, M.B., B.S. LOND., SURGICAL REGISTRAR, GUY'S HOSPITAL.

CertaIN joints in man possess interarticular fibrocartilages, namely, the knees, the temporo-mandibular articulations, the sterno-clavicular joints, and the joints between the bodies of the vertebræ. The triangular cartilage separating the inferior radio-ulnar from the wrist-joint is an additional instance. In each case the interarticular cartilage forms a more or less complete partition, producing a double joint, each part of which has its own distinct movement-a movement which (except in the case of the intervertebral joints) is of a different kind from that of the neighbouring part.

In the knee, on the femoral side of the interarticular cartilages, hinge motion of flexion and extension takes place; on the tibial side a slight degree of rotation is permitted during flexion. In the temporo-mandibular joint a gliding backwards and forwards takes place above the interarticular fibro-cartilage, whilst below the cartilage hinge movements of opening and closing the mouth are possible, together with some little rotation. In the sterno-clavicular joint up-and-down movements are said to occur on the clavicular side, and forward and backward movements on the sternal side of the interarticular cartilage. The triangular cartilage, uniting the radius to the styloid process of the ulna, by its upper surface axially rotates on the ulna during pronation and supination, but its lower surface helps to form the wrist-joint proper at which backward and forward or lateral movements can occur. The spine gains increased range of movement from the duplication of the intervertebral joints by the discs, but the kind of movement in adjacent synovial sacs is similar.

With the exception, therefore, of the intervertebral joints, the variety of movement in a joint is increased by the presence of interarticular cartilage. Should an interarticular cartilage lose its attachment, it is liable to shift its position and interfere with the mechanism of the joint. This is evidenced in the internal derangements of the knee and of the temporo-mandibular articulation. The other interarticular cartilages in man are so securely anchored as to resist detachment. The general rôle of interarticular cartilages has thus been outlined. In the special case of the knee certain points in their structure and function require closer examination.

The general shape of the cartilages is suggested by their name-semilunar. But there are two important points in their conformation which are less frequently emphasised. In the first place, they are wedge-shaped in section, with the apex pointing into the centre of the joint (vide Figs. 1, 2, 3, 4), and so, considering that both upper and lower surfaces are smooth and freely lubricated with synovial fluid, it is difficult to imagine that a normally lubricated, undetached cartilage should become severely squeezed between femur and tibia. In the second place, whilst the lower surface of each cartilage is flat, gliding easily on the fiat top of the tibia, the upper surface is concave, so that each semilunar cartilage forms a socket for one femoral condyle. The oval shape of 